\title{
Second US nurse with Ebola had traveled by plane
}

\author{
Michael McCarthy \\ Seattle
}

The second nurse to contract Ebola in the United States after caring for a Liberian man in a Texas hospital had flown on a commercial flight the day before she was hospitalized with the infection, US health officials said on 15 October.

The nurse, who has been identified in press reports as Amber Vinson, 29, was admitted to Texas Health Presbyterian Hospital in Dallas on 14 October after developing a fever.

In a press briefing Tom Frieden, director of the US Centers for Disease Control and Prevention (CDC), said that, because the nurse had recently been in contact with an Ebola patient, she should not have been on the aircraft. "The CDC guidance in this setting outlines the need for what is called controlled movement. That can include a chartered plane [or] a car, but it does not include public transport," Frieden said.

On the day of the flight Vinson reported a temperature of $99.5^{\circ} \mathrm{F}$ $\left(37.5^{\circ} \mathrm{C}\right)$, but unconfirmed press reports have said that she was nevertheless cleared to fly and returned home on a flight from Cleveland, Ohio to Dallas/Fort Worth in Texas. Frieden said that the chances of Vinson being contagious during the flight were "extremely low." Nevertheless, the CDC has asked all 132 passengers from the flight to contact the agency so that public health officials can interview them and arrange for follow-up. Those who are thought to be at risk of infection will be actively monitored.

Vinson is the second nurse at Texas Presbyterian Hospital to have had Ebola diagnosed. The first-Nina Pham, 26, of Fort Worth - entered the hospital on 10 October and is listed as being in good condition. Pham and Vinson had both taken care of Thomas Eric Duncan, a 42 year old Liberian man who contracted the infection while in west Africa but who did not develop symptoms until he arrived in Dallas in late September. He was admitted to the hospital on 28 September.

Frieden said, "Our investigations increasingly suggest that the first several days before the patient [Duncan] was diagnosed appear to be the highest risk period. That was the 28th, 29th, and 30th. These two healthcare workers both worked on those days and both had extensive contact with the patient when [he] had extensive production of body fluids because of vomiting and diarrhea." Duncan died on 8 October. ${ }^{1}$

The nurses' infections and the handling of Duncan's case have raised concerns that US hospitals are unprepared for handling Ebola patients. When Duncan first sought care on 25 September at Texas Presbyterian Hospital's emergency department complaining of fever, severe abdominal pain, and decreased urinary output, doctors diagnosed sinusitis and sent him home with antibiotics, even though he had told hospital staff that he had recently arrived from west Africa.

National Nurses United (NNU), a US nurses' union and professional organization, said that registered nurses at the hospital had reported that, when Duncan returned three days later, he was not placed in isolation immediately but was kept for several hours in an area where other patients were present and that his laboratory specimens were not given special handling. To protect the nurses from retaliation their names were not disclosed, the NNU said. It added in a statement, "There was no advance preparedness on what to do with the patient, there was no protocol, there was no system. The nurses were asked to call the Infectious Disease Department. The Infectious Disease Department did not have clear policies to provide either."

The nurses who initially took care of Duncan were not given impermeable gowns, wore gloves with no taping around the wrists, and had areas of their necks exposed, the statement said. "Were protocols breached? The nurses say there were no protocols," the NNU said.

And in a statement issued in response to the NNU's allegations, Texas Health Presbyterian Hospital said that it would review and respond to any concerns raised by its nurses and employees. "Patient and employee safety is our greatest priority and we take compliance very seriously," the hospital's statement said. In the press briefing, Frieden said that during the first several days of Duncan's stay-before Ebola was diagnosed-there was "a lot of variability in the use of personal protective gear" and some healthcare workers were "putting on three or four layers of protective equipment in the belief that this would be more protective." This was understandable, Frieden said, "but by putting on more layers of gloves or other protective clothing, it becomes harder to put them on and take them off"-raising the risk of contamination.

Dallas health authorities are monitoring 48 people in the community who had contact with Duncan before he was hospitalized, as well as one who had contact with Pham before she was isolated. None has developed symptoms. And about 50 additional Texas Presbyterian Hospital staff who entered Duncan's room during his hospitalizations are also being monitored for fever and other signs of infection.

After news of the second health worker's infection, President Barack Obama canceled a campaign trip and called a cabinet meeting to discuss the Ebola outbreak. In a brief press conference after the meeting, Obama said that he had directed the CDC to deploy a "rapid response team, a SWAT team, 
essentially, from the CDC to be on the ground as quickly as possible-hopefully within 24 hours-so that they are taking the local hospital step by step through exactly what needs to be done and making sure that all the protocols are properly observed."

He added, "The key thing to understand about this disease is that these protocols work. We know that because they've been used for decades now in Ebola cases around the world."

Obama said he was confident that US health officials could prevent a serious Ebola outbreak in the US but that it would become more difficult to do so if the outbreak in west Africa was not checked. "The single most important thing that we can do to prevent a more serious Ebola outbreak in this country is making sure that we get what is a raging epidemic right now in west Africa under control," he said.

Ebola virus disease is a viral hemorrhagic fever caused by a filovirus first discovered in Africa in 1976. Infection is thought to require direct contact with bodily fluids from a person who has developed symptoms or with contaminated objects such as syringes. On average, symptoms develop 8 to 10 days after exposure, but the incubation period can range from 2 to 21 days. People are not thought to be infectious during the incubation period. Symptoms include fever, weakness, severe headache, muscle and abdominal pain, vomiting and diarrhea, and unexplained bleeding and bruising.

The World Health Organization had reported 8997 confirmed, probable, or suspected cases of Ebola across seven affected countries as of 12 October: Guinea, Liberia, Nigeria, Senegal, Sierra Leone, Spain, and the US. It reported 4493 deaths.

Vinson, who is reported to be ill but in a stable condition, was transferred on Wednesday 15 October to Emory University Hospital in Atlanta, Georgia, which has one of the four high level isolation units in the US.

So far three Ebola patients have been treated at the Emory facility. Two of those patients-Kent Brantly, a physician, and Nancy Writebol, a nurse, who are both US missionaries-have been discharged. Both became infected while caring for Ebola patients in Liberia. A third patient, whose identity has not been disclosed, remains under treatment.

1 McCarthy M. Liberian man being treated for Ebola in Texas dies. BMJ 2014;349:g6145.

Cite this as: BMJ 2014;349:96277

๑ BMJ Publishing Group Ltd 2014 